\title{
Measurement and Preliminary Analysis of Powertrain Vibrations in Vehicle Production Environment
}

\author{
Andreas Manolatos, Ioannis Kagalidis, Branislav Vuksanovic \\ School of Engineering \\ University of Portsmouth \\ Portsmouth, UK \\ e-mail: andreas.manolatos@myport.ac.uk
}

\begin{abstract}
In a vehicle production environment, obtaining information on the condition of the assembled vehicle, allows for increased quality while minimizing rework minutes at the same time. The cost of this information is associated with additional time in production, increasing the overall cost of the vehicle. The focus of this paper is development of a system for contactless measurement and investigation of vibrations present in the vehicle and the Rolling Road vehicle testing facility as a mean of identifying failures or degradation in the vehicle or testing facility. Measurements are performed using a contactless Laser Vibrometer based system which facilitates capturing the vibrations without interfering with the vehicle and other standard testing procedures. Signal conditioning and analysis methods are applied to measured vibration signals in an attempt to detect irregularities and determine their transient characteristics from the captured signals. Data analysis techniques employed in this initial trial of the system include Short Time Fourier and Wavelet Transforms. Proposed testing method including experimental setup, captured signals and some preliminary analysis results are discussed in this paper.
\end{abstract}

Keywords-signal processing; vibration analysis, nonstationary signals; laser vibrometry; wavelet analysis

\section{INTRODUCTION}

In a vehicle production environment, the need for faster, more accurate and more efficient means of testing is of paramount importance. While the vehicle is expected to be tested thoroughly in each of the individual stages of assembly, there are still ways of obtaining additional information by detecting, monitoring and subsequently analysing the auditory and vibration information provided by the vehicle itself when executing the first full engine test-run during the production stage and before it is eventually released to the end customer. Detailed analysis of these signals can result in a significantly better overview of the vehicle and help in identifying potential problems related to the vehicle. Valuable additional information about the vehicle as a whole or individual sub-systems and modules can be gained. This includes the engine, the exhaust system, the horn, the vehicle sound generator in hybrid variants as well as the infrastructure and the Rolling Road facility.

Vibration analysis is becoming a widely used tool in diagnosis of various machine faults, including bearings, gears and similar equipment. Gearboxes in wind turbines, automobiles and helicopters often run at time varying speeds and/or loads. This operation gives rise to a non-stationary vibration signals which can be monitored and further analysed to provide more information about the condition of the gearbox. Bafroui et al. [1] have investigated and reported the use of wavelet energy and Shannon entropy measures for feature extraction and detection of faults in gearboxes. Chen et al. [2] carried out fault feature extraction for gearboxes under non-stationary conditions during the run-up or rundown process. During the run-up or run-down periods gear drive stimulation forces change both in amplitude and in frequency allowing some events and phenomena to become more obvious under the varying speed conditions, usually not evident at constant speed operation.

Vibration signals have been extensively utilized since they provide the most intrinsic information about mechanical faults [3]. Common vibration sensors include piezoelectric and MEMS accelerometers, displacement as well as velocity sensors which are all widely used in bearing fault detection of the gearbox in various industrial applications [4], [5].

In many practical applications attaching the sensory element (acoustic sensor or accelerometer) directly on the rotating parts is impractical, can affect the operating conditions of the device under test and is not cost-effective. This can also be the case in industry or harsh working environments which presents a challenge in distinguishing and extracting signal characteristics [6]-[8].

The focus of this work is detection, identification and prediction of a vehicle and to a certain extent, testing infrastructure acoustic emission and vibration during the production stages. Very limited amount of research has been attempted to address these challenges and topics directly in the production environment.

This paper details design and testing process of the vibration measurement and recording system and discusses the signal processing techniques used to condition the measured signal and extract the useful information from it. The measured signal contains the information about vibrations originating from a moving components and is of the analogue nature, thus the first step includes conversion of the captured signal to its discrete version. According to their statistical properties, signals emanating from the measuring equipment and the vehicle itself and any faults or deviations from the expected performance, can be further considered to be of non-stationary nature, i.e. statistical properties of those 
signals are expected to have time-variable statistical properties [9]. This property would make direct application of the most common signal analysis tool - Fourier Transform (FT) unreliable. FT would in most cases not be able to determine the transient signal characteristics as the results will reflect the spectral content of the measured signal averaged through the duration of the signal [10].

Time-frequency analysis techniques, such are Short Time Fourier (STFT) and Discrete Wavelet Transform (DWT) have therefore been used in this work instead of FT. Those techniques allow for better signal representation in both the frequency and time domains which makes them more suitable for the analysis of non-stationary type signals. Furthermore, these methods provide more information of the energy distribution over the frequency bands [11].

The rest of this paper is organized in the following manner. Section II describes the experimental setup developed in order to measure and record vehicle vibration signals. Main elements of the system - sensors, conditioning circuitry and recording facility are discussed and the reasons for this choice justified. Section III contains some preliminary results obtained during the system testing and validating process. Signals captured during the vehicle tests and processing of those signals are explained and results illustrated indicating the potential use of developed system. Conclusions and further steps in this project are outlined in the final, Section IV of the paper.

\section{MEASUREMENT SYSTEM}

\section{A. Sensor and Associated Circuitry}

In order to measure the vibration from the product under the test, a Laser Vibrometer system was designed specifically for this application. Designed system is recording the vibration information carried by a visible light laser beam reflected off the target surface. Schematic of the circuit and a physical 3D image of the measurement device developed for this experiment are shown in Figure 1.

The device's main components are an active photosensitive element and a small amplifier to increase the combined signal fed to the audio input of a computer system for further processing. As it is aimed as a stand-alone device, a $9 \mathrm{~V}$ battery is used to power the circuit and a variable gain is added for fine tuning and calibration. Due to the external and environmental operating conditions, an additional lownoise pre-amplification stage was used between the active photosensitive element and the final stage amplifier, to increase the driving potential of the received signal as well. To further secure the device from unwanted noise sources, high-precision components, short track lengths and an insulated casing was used while the remaining noise components are filtered out using digital filters implemented in software.

The photosensitive element of the device is a visible light phototransistor that offers high reaction speeds, very low noise characteristics and low-effort alignment. Gain and sensitivity settings can be independently configured for optimized performance of the device and increased operating range.

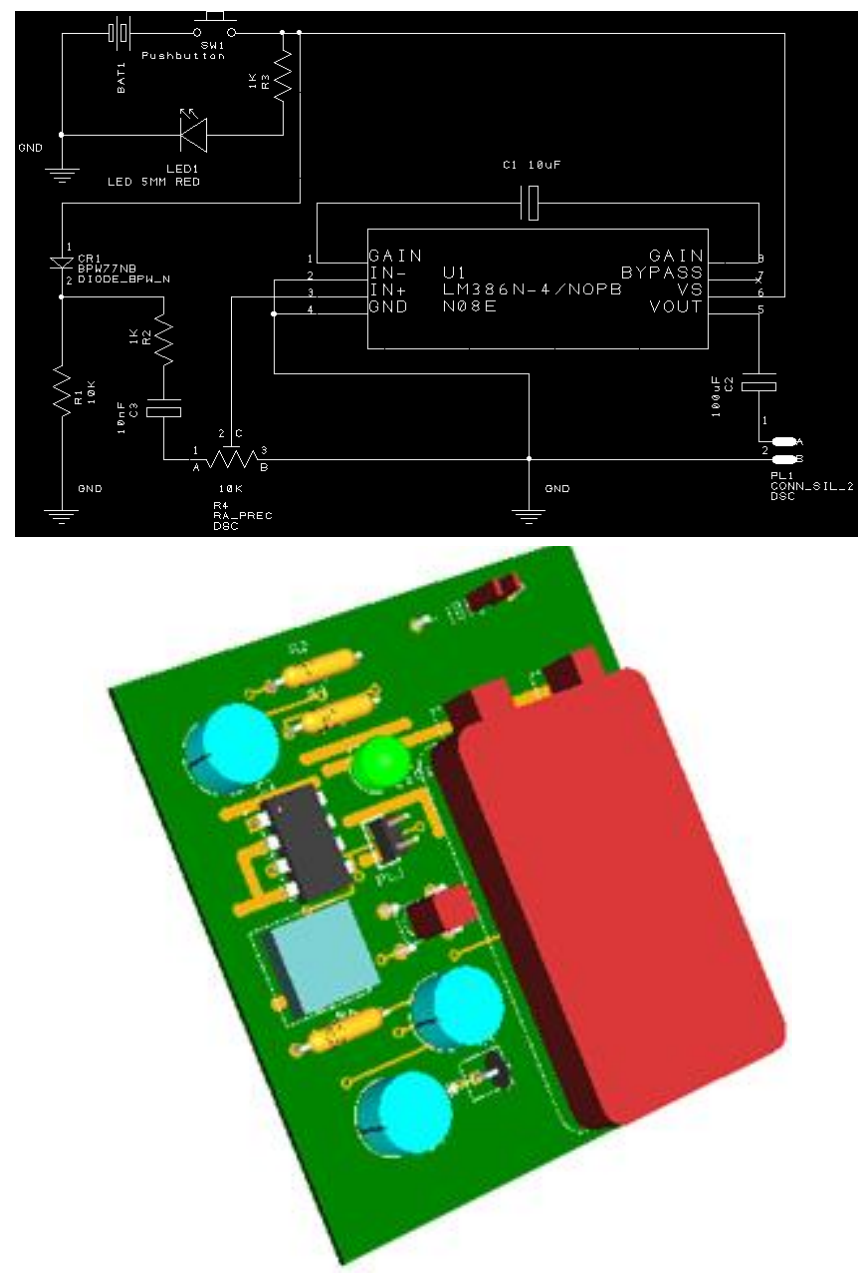

Figure 1. Laser vibrometer circuit and 3D image

The interface of the device is a standard $3,5 \mathrm{~mm}$ audio interface, which allows easy connectivity with computer terminal and loudspeaker isolation when the audio interface was connected. Finally, a red-tinted focusing lens was used to improve focusing of the incoming laser beam.

The signals obtained through the measurement procedure contain features and components that can be attributed to the vehicle itself, the testing equipment used in the measurement process and infrastructure. In addition to this, signals can also be affected by the noise from the surrounding environment and parasitic interference from the enclosure of the testing facilities. The desired information or the components related to condition of the vehicle under test as well as the testing facility itself is polluted by the environmental and the valuable information will have to be extracted from the measured signal through further processing of the captured signal in discrete-time domain.

\section{B. Experimental Setup}

An initial trial of the system involved measuring the vibration and internal noises of 11 vehicles during actual testing in the Rolling Road facility. A low power ( $1 \mathrm{~mW})$ Class $2650 \mathrm{~nm}$ (red) laser was used as the targeting beam, 
which is the maximum commercially allowed laser device. A reflective surface was used to reflect the laser beam with the vibrometer positioned $1.5 \mathrm{~m}$ from the target point, at a $45-$ degree angle as indicated in Figure 2.

The vibrometer had the lens, the gain and sensitivity adjusted to compensate for the ambient and environmental noise. It was placed on different surface materials and on flex bases to dampen any other vibrations that could pollute the results of the trial using telescopic tripods to mount both the vibrometer and the laser device. Each test signal is approximately 2 minutes long in duration, depending on the vehicle options and the driver's speed in carrying out the test in the Rolling Road facility.

The system was tested using an omnidirectional microphone as a reference sensor in order to compare acoustic data captured with the microphone to vibration data taken by the vibrometer. Sample results - spectrograms of the signals captured by two sensors are shown in Figure 3. Spectrogram represents a spectrum of frequencies present in the measured signal as they vary within the measured time frame and is a suitable tool for the visual inspection of this type of measured data.

Comparing the two spectrograms recorded from both the microphone and the vibrometer, it is apparent that there are no audio or vibration data from the microphone signal in the $0-900 \mathrm{~Hz}$ range. The microphone signal seems to contain useful information from $1000 \mathrm{~Hz}$ and above with significantly higher content of information.

The vehicle RPM acceleration curve can be seen in both signals with the microphone performing better on the higher frequencies and the vibrometer in the lower frequencies as expected with a clear indication in the $100-3000 \mathrm{~Hz}$ range that the vibration information can be detected. Low frequency range between $0-1 \mathrm{kHz}$ is the spectral content of vehicle vibrations not detected by the microphone. The aim of the further work on this project is to try and use this portion of spectral information in order to try and identify and predict upcoming problems with the vehicle or the facility.

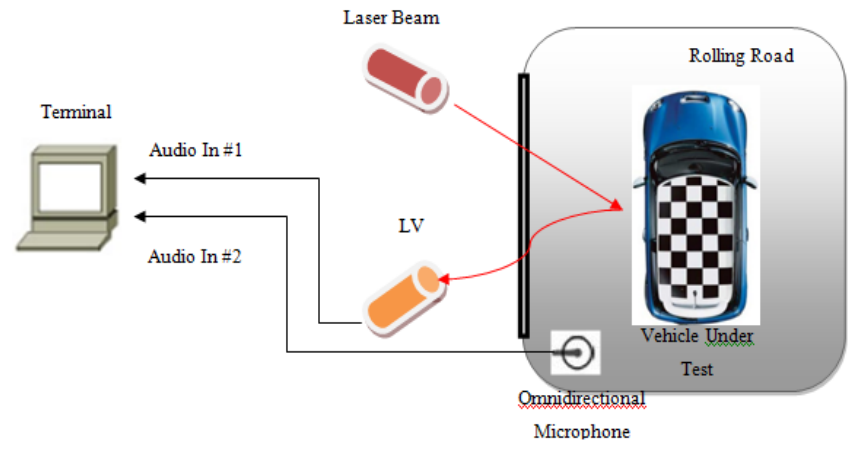

Figure 2. Experimental setup

It is clear that the vibrations increase with the increase in the vehicle acceleration and differences in those patterns should be further analysed to give more information about the condition of the facility or the vehicle under test.
Another trial to compare the performance of the system at a longer distance $(3 \mathrm{~m})$ and different angles $(30-60$ degrees) was performed with similar results.

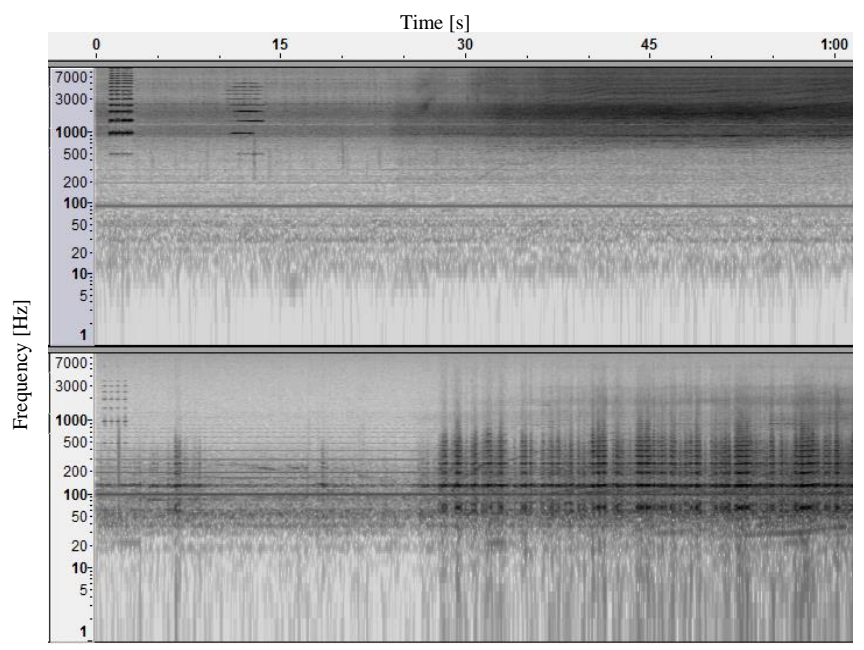

Figure 3. Microphone and vibrometer data comparison

\section{Measurement Process and Data Collection}

The data were gathered in Rolling Road facility $\mathrm{C}$ in the BMW Plant Oxford using the experimental setup described in the previous section thus allowing access to real data in everyday working conditions. The possible contaminating sources have been identified as the adjacent Rolling Road facilities and the ambient or environmental sounds from the production area. The rest of the infrastructure and equipment, in regards to the status and faults of the vehicle after testing and the facility itself, was already configured and calibrated.

The data capturing equipment was installed outside the facility to avoid any common mode errors from the vibrations or industrial equipment interference using telescopic tripods to mount and stabilise both the laser beam and the data capturing circuit. The beam was aimed through the double paned window of the facility and focused on providing the optimum reflection pattern. A laptop was used to record the captured in the real-time and a microphone was placed inside the facility to record the audio signal of the facility and the vehicle under test as additional information.

The driver was bringing the car into the facility and was performing the standard set of tests and operations - scan the vehicle identification number, activate horn, start the test, follow on screen instruction - including pre-warming drive curve if the engine temperature is low, speed ramp-up with progressive gear changes, turbocharger adaption and repeat on failure, variable valve flushing etc. depending on the vehicle series, options and engine type - and drive off. Each test took a different time depending on the content of the test and vehicle itself.

The vibrations from the vehicle were first detected when a vehicle was in the facility and the current speed was 26 $\mathrm{km} / \mathrm{h}$. The best performance was measured when the speed was $80+\mathrm{km} / \mathrm{h}$. During these recording sessions, not all vehicles were successfully captured due to misalignments of the beam or the focusing lens on the vibrometer. 
In turn, each result was further processed, correlated to the microphone signal and stored in an attempt to determine the optimum setup and data gathering process.

\section{DATA ANALYSIS}

The aim of the preliminary data analysis is to establish whether the additional data about the condition of the vehicle and testing facility can be detected while the vehicle is under test by using the Laser Vibrometer based contactless method for data gathering. The vibration data gathered from the system were classified in two groups discussed in the rest of this section for further analysis.

\section{A. Horn Detection Results}

The horn of the vehicle is located in the engine compartment and is powerful enough to generate vibrations that can be detected with the existing setup. Cars under the test can have one of two different types of horns - the high, $500 \mathrm{~Hz}$ and low $400 \mathrm{~Hz}$ frequency variant including +/- 25 $\mathrm{Hz}$ tolerance for both types.

The aim of this test is to merely establish whether the measurement system and captured vibration signals can be used to detect and differentiate between the two different horn types. Due to the noise contamination of the measurement area from adjacent lines and vehicles under the test activating their horns at the same time, the direct measurement of the horn vibration from the vehicle can ensure that the target vehicle is monitored and not an adjacent one.

The sample horn test data shown in Figure 4 include a time-synchronised comparison of the recorded data from the measurement system targeting a production vehicle during the Rolling Road test. The measurement lasts between $1-2$ seconds and the test requires the activation of the horn four times. This test is performed by the driver in order to validate the functionality of the horn.

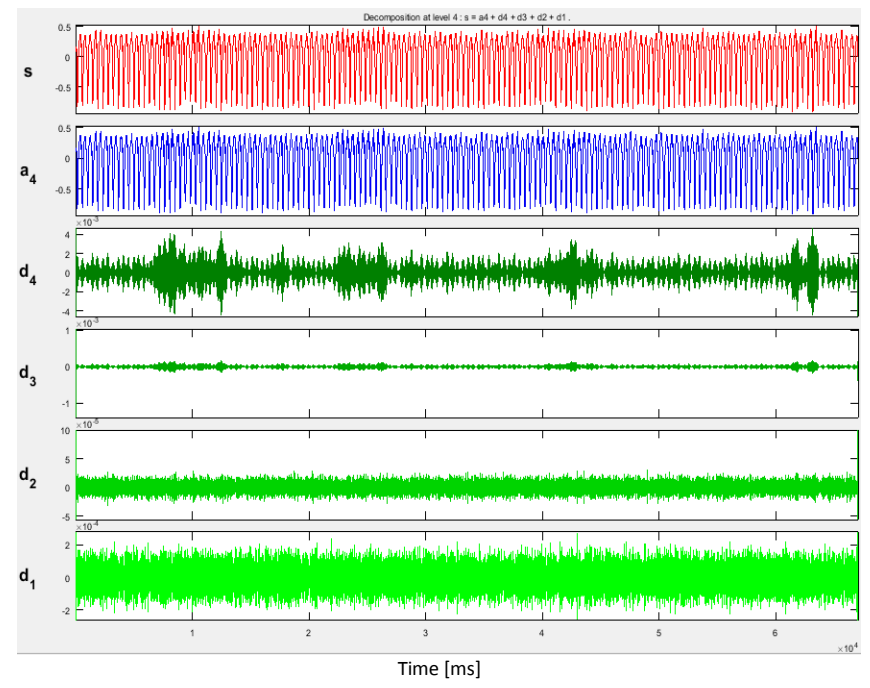

Figure 4. Horn detection signal analysis

The first plot shows the recorded vibration data after normalization, recorded live from the vehicle and contains four horn activations. Horn activation instants are hard to detect by visual inspection of this signal.

The second plot on this figure contains the STFT spectrogram of the original normalized waveform. This analysis shows more information on the frequency content of the recorded signal with the four horn activations centered at $495 \mathrm{~Hz}$ but it is not clear where each activation starts and finishes.

Furthermore, the fourth activation can be lost in the background noise level as the recorded level was lower than the rest of the activations.

The graphs below the spectrogram show the original signal decomposition at 4 levels using a db4 wavelet after performing a DWT and identifying this as the optimum level of decomposition. The $\mathrm{S}$ graph is the original signal followed by the relevant approximation (a4) on the $4^{\text {th }}$ level, resulting in an almost exact reconstruction of the original signal. The next graph contains the $4^{\text {th }}$ level decomposition detail (d4) which show very clearly the point of each horn activation (119ms / 432ms / 824ms / 1337ms) and the relative time duration of each one, effectively detecting these activations as discontinuities and irregularities of the original signal regardless of the noise level present in the original normalized recording. The $3^{\text {rd }}$ level decomposition detail contains the same information but there is an irregularity detected in the beginning of the signal changing the scale of the detail result.

The last two detail levels contain vibration and noise information but no usable information can be extracted from them.

\section{B. Drive Curve Comparison and Gear Shift Detection}

DWT based approach described in the previous section has been used for the second set of tests aimed at evaluating the measurement procedure and recorded data. The aim is to use vibration signals to try and identify when and how the gear shift changes occur while the vehicle is driven in the Rolling Road for the first time. During this test the vehicle does the first full driving cycle after it has been assembled, thus this is the first time where the vehicle is tested in driving conditions. The testing process involves the majority of the powertrain checks by doing a warm-up drive cycle if the engine temperature is low and then follows a drive up curve that gradually increases the speed of the vehicle while sequentially changing gears, the turbocharger adaption and a repeat in the drive curve if the adaption has failed. Variable valve timing check and flushing, braking and drive-out curve are performed at the end of each test.

The driving curve has a variable testing time which depends on how closely each driver follows the on-screen instructions, what is the vehicle type and what are the installed options. On average, this test is expected to last approximately 90 seconds assuming the adherence to the instructions by the driver, no warm-up cycle and no repeated test steps. During this drive cycle, the measured vibrations include the ones coming from the vehicle and its vibrating components as well as the vibrations coming from the testing facility itself, even though the target of the vibrometer is the vehicle under test. Direct separation of those vibrations using 
a single vibrometer is not possible as both the vehicle and the facility have to be monitored to identify the possible source of the vibrations. This fact should be kept in mind while using the recorded signal, the sum of those vibrations, to identify problems that appear during the testing process. Given the fact that there is a reference drive curve along with the expected steps to be followed, the measured vibrations can be correlated to identify any discrepancies that highlight a possible problem either on the vehicle or on the facilities side. Problems of this type are characterized by abrupt changes or degradation in the transient features of the expected signal.

The mechanical stress on the powertrain happens during gear shift changes as most of the powertrain components are engaged at the same time to maintain an optimum performance. The same stress can be propagated to the testing facility and serve as a vibration trigger on the facility's side and vice versa.

Sample signal recorded during this drive test and the results of the processing are shown in Figure 5. Drive curve identifying various events during the test is superimposed at the top of this figure to provide easier identification of various events during this test. The first plot below the drive curve shows the recorded vibration data after normalization as it was recorded live from the vehicle and includes the start of the drive test until the vehicle comes to a standstill just prior to the drive off. Again, no information in regards to the condition of either the vehicle or the facility can be extracted by this waveform as it is expected.

The second plot from Figure 5 contains the STFT spectrogram of the original normalized waveform but this time the frequency information can be seen and compared to the actual drive curve of the vehicle. The spectrogram deliberately shows information up to $1500 \mathrm{~Hz}$ to include and focus on the low frequency vibrations and any harmonics present. Visual inspection of the spectrogram outlines the changes in the vehicle speed during the drive curve, starting from $0 \mathrm{~km} / \mathrm{h}$, gradually increasing until $150 \mathrm{~km} / \mathrm{h}$ and remaining there for a small period of time and gradually reducing speed until the vehicle comes to a standstill again prior to driving out. This information can be used when compared with the reference drive curve to detect any anomalies on its own, to identify deviations from the reference drive curve and to indicate possible problems with either the vehicle or the testing facility.

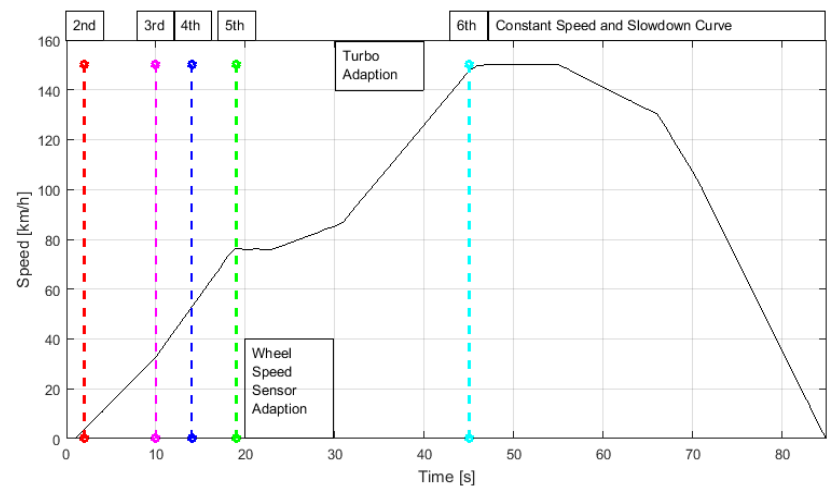

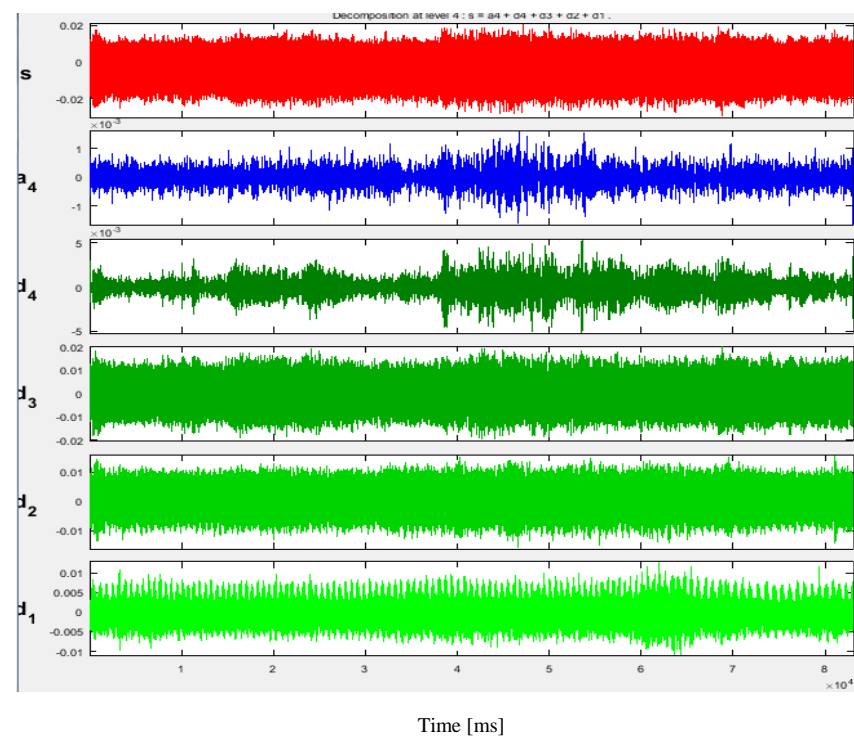

Figure 5. Gear-shift detection results

Next plot on this figure contains the results of the wavelet analysis of the same signal. The signal was decomposed at 4 levels using a db4 wavelet after performing a DWT and identifying this as the optimum level of decomposition. The $\mathrm{S}$ graph is the original signal followed by the relevant approximation (a4) on the $4^{\text {th }}$ level, resulting in an almost exact reconstruction of the original signal.

The level 4 detail (d4) holds the most interesting information, where individual irregularities are highlighted by the wavelet analysis. Each highlight covers a band that happens during a gear shift change when compared to the reference drive curve. Each band now, in turn, indicate the transient characteristics of the vibrations governing each gear change that can be used further to identify any deviations that can be translated in problems either in the vehicle or in the testing facility. It is also worth noting that another interesting area of analysis is the steady speed point, where the vibrations are stronger due to the increased speed and the slowdown roll-off curve where breaking can trigger different vibrations from other vehicle components such as brake calipers and suspension mechanisms which can also be used to monitor any upcoming problems.

The rest of the details $(\mathrm{d} 1-\mathrm{d} 3)$ contain vibration and noise information but no usable information can be extracted from them.

\section{Conclusions AND THE NeXT STEPS IN THE PROJECT}

The initial steps in setting up and testing a novel system for the vibration analysis in a vehicle commissioning and testing environment have been described in this paper. The necessary hardware including the measurement sensors, signal capturing and conditioning circuitry as well as signal recording facility have been designed and trialed. A number of vehicles assembled at the BMW plant in Oxford have been tested using designed system and their vibration signatures recorded and analysed. The preliminary analysis of the captured signals focused on establishing the clear 
correlation between the recorded signal and the vehicle vibrations in the vehicle testing and commissioning environment. Recorded raw signals have been processed using various digital signal processing (DSP) techniques in order to reduce the level of noise and extract the most important signal components which can be reliably recognized and interpreted.

The use and effectiveness of wavelet analysis for this task has been highlighted by comparing the results of the DWT and STFT analysis methods with the raw signals. The wavelet properties allow for an accurate recognition of timing information in the signal as well as extraction of irregularities and transient characteristics which can then be used to identify existing problems or even pinpoint degradation in these elements that can prompt for preventive maintenance if needed.

Concerning the measurement system, the drawback of a purely acoustical measurements, i.e. microphone-only approach have been exposed as the additional and unwanted information from the adjacent facilities where sounds from other vehicles running the same test at the same time contaminate the measured signal. This was demonstrated by the second horn signal being detected in the initial horn test measurements on the vehicle. The vibrometer based system, on the other hand, while recording only the noise emanating from the vehicle under the test and test facility has a potential problem of not being able to detect higher frequency content present in the signal. One of the immediate tasks in the continuation of this work is to establish how important is the higher frequency information in providing additional information on the condition of the vehicle and the facility. The ideal approach might therefore be to combine the data captured by both sensors in order to achieve a more holistic approach. The need to adopt this approach is highlighted by the fact that detection reliability of the current system is below $99 \%$.

On a broader scale, this work should aim for the system that can be used on various sites and plants of vehicle production by adapting to the individual characteristics of each environment. In addition to this the possibility of an onboard solution that can supplement the existing on-board diagnostics of a vehicle by analyzing the vibrations present on the vehicle.

With those, broader aims and targets in mind the future work will focus on investigating the advanced techniques for signal processing and analysis. Possible algorithms and methods to investigate will include the analysis of the acquired vibration signal using ensemble empirical mode decomposition (EEMD) and singular spectrum analysis
(SSA) methods for signal decomposition, denoising and feature extraction. Adaptive Noise Cancellation (ANC) can also be applied to try and reduce the unwanted noise present in the measurements.

Further study would then include the use of data mining techniques as well as pattern recognition and prediction algorithms such as Artificial Neural Networks (ANN) and Independent Component Analysis (ICA) in the context of vehicle testing in production environments which might also be applicable to an area such as the automotive industry.

\section{REFERENCES}

[1] Hojat Heidari Bafroui, Abdolreza Ohadi, Application of wavelet energy and Shannon entropy for feature extraction in gearbox fault detection under varying speed conditions, Neurocomputing, Volume 133, 10 June 2014, Pages 437-445, ISSN 0925-2312

[2] X. Chen, Z. Feng and M. Liang, "Fault feature extraction of planetary gearboxes under nonstationary conditions based on reassigned wavelet scalogram," 2015 IEEE International Instrumentation and Measurement Technology Conference (I2MTC) Proceedings, Pisa, 2015, pp. 294-299.

[3] Y. Lei, F. Jia, J. Lin, S. Xing and S. X. Ding, "An Intelligent Fault Diagnosis Method Using Unsupervised Feature Learning Towards Mechanical Big Data," in IEEE Transactions on Industrial Electronics, vol. 63, no. 5, pp. 3137-3147, May 2016.

[4] H. Zhang, Q. Yue, H. Zhou, J. Si and X. Shi, "Automatic selection of frequency-band based on wavelet for gear fault diagnosis," Computer Science \& Education (ICCSE), 2015 10th International Conference on, Cambridge, 2015, pp. 524-527.

[5] Y. Qu, J. Zhu, D. He, B. Qiu and E. Bechhoefer, "Development of a new acoustic emission based fault diagnosis tool for gearbox," Prognostics and Health Management (PHM), 2013 IEEE Conference on, Gaithersburg, MD, 2013, pp. 1-9.

[6] V. B. Pagi, R. S. Wadawadagi and B. S. Anami, "An acoustic approach for multiple fault diagnosis in motorcycles," SoftComputing and Networks Security (ICSNS), 2015 International Conference on, Coimbatore, 2015, pp. 1-7.

[7] H. V. Khang, H. R. Karimi and K. G. Robbersmyr, "Bearing fault detection based on time-frequency representations of vibration signals," Electrical Machines and Systems (ICEMS), 2015 18th International Conference on, Pattaya, 2015, pp. 1970-1975.

[8] S. H. Kia, H. Henao and G. A. Capolino, "A real-time platform dedicated to on-line gear tooth surface damage fault detection in induction machines," Electrical Machines (ICEM), 2014 International Conference on, Berlin, 2014, pp. 1478-1484.

[9] M. R. Wilkinson, "Condition monitoring for offshore wind turbines "Eng. D., University of Newcastle upon Tyne, UK, 2008

[10] T. Sawicki, A. K. Sen, and G. Litak, "Multiresolution wavelet analysis of the dynamics of a cracked rotor," International Journal of Rotating Machinery, vol. 2009

[11] A. A. Jaber and R. Bicker, "A Simulation of Non-Stationary Signal Analysis Using Wavelet Transform Based on LabVIEW and Matlab," Modelling Symposium (EMS), 2014 European Pisa, pp. 138-144 